\title{
ON SUMMABILITY OF NONLINEAR MAPPINGS: A NEW APPROACH
}

\author{
DANIEL PELLEGRINO AND JOEDSON SANTOS
}

\begin{abstract}
The main goal of this paper is to characterize arbitrary nonlinear (non-multilinear) mappings $f: X_{1} \times \cdots \times X_{n} \rightarrow Y$ between Banach spaces that satisfy a quite natural Pietsch Domination-type theorem around a given point $\left(a_{1}, \ldots, a_{n}\right) \in X_{1} \times \cdots \times X_{n}$. As a consequence of our approach a notion of weighted summability arises naturally, which may be an interesting topic for further investigation.
\end{abstract}

\section{INTRODUCTION}

The theory of absolutely summing operators was initiated with Grothendieck's ideas in the 50s but just in the sixties (see [19, 28]) the results were better understood and fully explored (for details we refer to the book [14]). Besides its intrinsic interest, this theory has beautiful applications in Banach space theory and nice connections with the geometry of the Banach spaces involved (see, for example, [8, 19] or [7] for a more recent approach). Due to the success of the linear theory, it is not a surprise that many authors have devoted their interest to the nonlinear setting; the multilinear theory, however, has a longer history, which seems to start with [3, 20]; for recent different nonlinear approaches and applications we mention [10, 11, 12, 13, 18, 22, 23, 24, 26, 27] and references therein.

Pietsch Domination-Factorization Theorems play a central role in the theory of absolutely summing linear operators and provide an unexpected and beautiful measure theoretic taste in the theory (for details we mention the monographs [2, 9, 14, 30]). In the last decade several different nonlinear versions of Pietsch Domination-Factorization Theorem have appeared in the literature (see, for example, 11, 4, [5, 15, 16, 21]); for this reason, in [6], an abstract unified approach to Pietsch-type results was presented as an attempt to show that all the known Pietsch-type theorems were particular cases of a unified general version. The main problem investigated in the present paper is motivated by the PietschDomination Theorem (PDT) for $n$-linear mappings between Banach spaces, which we describe below.

From now on, if $X_{1}, \ldots, X_{n}, Y$ are Banach spaces over a fixed scalar field which can be either $\mathbb{K}=\mathbb{R}$ or $\mathbb{C}, \operatorname{Map}\left(X_{1}, \ldots, X_{n} ; Y\right)$ will denote the set of all arbitrary mappings from $X_{1} \times \cdots \times X_{n}$ to $Y$ (no assumption is necessary). The topological dual of a Banach space $X$ will be denoted by $X^{*}$ and its closed unit ball will be represented by $B_{X^{*}}$, with the weak-star topology.

D. Pellegrino by INCT-Matemática, PROCAD-NF Capes, CNPq Grant 620108/2008-8 (Ed. Casadinho) and CNPq Grant 301237/2009-3. 
Let $0<p_{1}, \ldots, p_{n}<\infty$ and $1 / p=\sum_{j=1}^{n} 1 / p_{j}$. An $n$-linear mapping $T: X_{1} \times \cdots \times X_{n} \rightarrow Y$ is $\left(p_{1}, \ldots, p_{n}\right)$-dominated if there is a constant $C>0$ so that

$$
\left(\sum_{j=1}^{m}\left\|T\left(x_{j}^{(1)}, \ldots, x_{j}^{(n)}\right)\right\|^{p}\right)^{\frac{1}{p}} \leq C \prod_{k=1}^{n} \sup _{\varphi \in B_{X_{k}^{*}}}\left(\sum_{j=1}^{m}\left|\varphi\left(x_{j}^{(k)}\right)\right|^{p_{k}}\right)^{1 / p_{k}},
$$

regardless of the choice of the positive integer $m, x_{j}^{(k)} \in X_{k}, k=1, \ldots, n$ and $j=1, \ldots, m$. The folkloric PDT for $\left(p_{1}, \ldots, p_{n}\right)$-dominated multilinear mappings (see [16] or [25] for a detailed proof) asserts that $T$ is $\left(p_{1}, \ldots, p_{n}\right)$-dominated if and only if there are Borel probabilities $\mu_{k}$ on $B_{X_{k}^{*}}, k=1, \ldots, n$, and a constant $C>0$ such that

$$
\left\|T\left(x^{(1)}, \ldots, x^{(n)}\right)\right\| \leq C\left(\int_{B_{X_{1}^{*}}}\left|\varphi\left(x^{(1)}\right)\right|^{p_{1}} d \mu_{1}\right)^{\frac{1}{p_{1}}} \cdots\left(\int_{B_{X_{n}^{*}}}\left|\varphi\left(x^{(n)}\right)\right|^{p_{n}} d \mu_{k}\right)^{\frac{1}{p_{n}}}
$$

for all $x^{(j)} \in X_{j}, j=1, \ldots, n$.

A related question, not covered by the abstract approach presented in [6], arises:

Problem 1.1. If $\left(a_{1}, \ldots, a_{n}\right) \in X_{1} \times \cdots \times X_{n}$, what kind of mappings $f \in \operatorname{Map}\left(X_{1}, \ldots, X_{n} ; Y\right)$ satisfy, for some $C>0$ and Borel probabilities $\mu_{k}$ on $B_{X_{k}^{*}}, k=1, \ldots, n$, the inequality

$$
\left\|f\left(a_{1}+x^{(1)}, \ldots, a_{n}+x^{(n)}\right)-f\left(a_{1}, \ldots, a_{n}\right)\right\| \leq C \prod_{k=1}^{n}\left(\int_{B_{X_{k}^{*}}}\left|\varphi\left(x^{(k)}\right)\right|^{p_{k}} d \mu_{k}\right)^{\frac{1}{p_{k}}}
$$

for all $x^{(j)} \in X_{j}, j=1, \ldots, n ?$

In the next section we solve Problem 1.1.

\section{Main Result}

Let $0<p_{1}, \ldots, p_{n}<\infty$ and $1 / p=\sum_{j=1}^{n} 1 / p_{j}$. We will say that $f \in \operatorname{Map}\left(X_{1}, \ldots, X_{n} ; Y\right)$ is $\left(p_{1}, \ldots, p_{n}\right)$ dominated at $\left(a_{1}, \ldots, a_{n}\right) \in X_{1} \times \cdots \times X_{n}$ if there is a $C>0$ and there are Borel probabilities $\mu_{k}$ on $B_{X_{k}^{*}}, k=1, \ldots, n$, such that (1.3) is valid for all $x^{(j)} \in X_{j}, j=1, \ldots, n$.

It is worth mentioning that Pietsch's original proof of his domination theorem uses Ky Fan Lemma instead of the usual Hahn-Banach separation theorem (see [29]). The use of Hahn-Banach theorem seems to be not adequate for proving our main result; for this task Pietsch's original idea of using Ky Fan Lemma will be very useful. It is in some sense a nice surprise that Pietsch's first argument conceived for linear maps has shown to be the more adequate when dealing with a very general and fully nonlinear context.

Lemma 2.1 (Ky Fan). Let $K$ be a compact Hausdorff topological space and $\mathcal{F}$ be a concave family of functions $f: K \rightarrow \mathbb{R}$ which are convex and lower semicontinuous. If for each $f \in \mathcal{F}$ there is a $x_{f} \in K$ so that $f\left(x_{f}\right) \leq 0$, then there is a $x_{0} \in K$ such that $f\left(x_{0}\right) \leq 0$ for every $f \in \mathcal{F}$. 
For the proof of our main theorem we will need the following lemma (see [17, Page 17]):

Lemma 2.2. Let $0<p_{1}, \ldots, p_{n}, p<\infty$ be so that $1 / p=\sum_{j=1}^{n} 1 / p_{j}$. Then

$$
\frac{1}{p} \prod_{j=1}^{n} q_{j}^{p} \leq \sum_{j=1}^{n} \frac{1}{p_{j}} q_{j}^{p_{j}}
$$

regardless of the choices of $q_{1}, . ., q_{n} \geq 0$.

Theorem 2.3. A map $f \in \operatorname{Map}\left(X_{1}, \ldots, X_{n} ; Y\right)$ is $\left(p_{1}, \ldots, p_{n}\right)$-dominated at $\left(a_{1}, \ldots, a_{n}\right) \in X_{1} \times \cdots \times X_{n}$ if and only if there is a $C>0$ such that

$$
\begin{aligned}
& \left(\sum_{j=1}^{m}\left(\left|b_{j}^{(1)} \ldots b_{j}^{(n)}\right|\left\|f\left(a_{1}+x_{j}^{(1)}, \ldots, a_{n}+x_{j}^{(n)}\right)-f\left(a_{1}, \ldots, a_{n}\right)\right\|\right)^{p}\right)^{1 / p} \\
& \leq C \prod_{k=1}^{n} \sup _{\varphi \in B_{X_{k}^{*}}}\left(\sum_{j=1}^{m}\left(\left|b_{j}^{(k)}\right|\left|\varphi\left(x_{j}^{(k)}\right)\right|\right)^{p_{k}}\right)^{1 / p_{k}}
\end{aligned}
$$

for every positive integer $m,\left(x_{j}^{(k)}, b_{j}^{(k)}\right) \in X_{k} \times \mathbb{K}$, with $(j, k) \in\{1, \ldots, m\} \times\{1, \ldots, n\}$.

Proof. In order to simplify notation, from now on we will write

$$
f\left(b^{(k)}, x^{(k)}\right)_{k=1}^{n}:=\left(\left|b^{(1)} \ldots b^{(n)}\right|\left\|f\left(a_{1}+x^{(1)}, \ldots, a_{n}+x^{(n)}\right)-f\left(a_{1}, \ldots, a_{n}\right)\right\|\right)^{p} .
$$

Assume the existence of such measures $\mu_{1}, \ldots, \mu_{n}$ satisfying (1.3). Then, given $m \in \mathbb{N}, x_{j}^{(l)} \in E_{l}$ and $b_{j}^{(l)} \in \mathbb{K}$, with $(j, l) \in\{1, \ldots, m\} \times\{1, \ldots, n\}$, we have, using Hölder Inequality,

$$
\begin{aligned}
& \sum_{j=1}^{m} f\left(b_{j}^{(k)}, x_{j}^{(k)}\right)_{k=1}^{n} \leq C^{p} \sum_{j=1}^{m} \prod_{k=1}^{n}\left(\int_{B_{X_{k}^{*}}}\left(\left|b_{j}^{(k)}\right|\left|\varphi\left(x_{j}^{(k)}\right)\right|\right)^{p_{k}} d \mu_{k}\right)^{\frac{p}{p_{k}}} \\
& \leq C^{p} \prod_{k=1}^{n}\left(\sum_{j=1}^{m} \int_{B_{X_{k}^{*}}}\left(\left|b_{j}^{(k)}\right|\left|\varphi\left(x_{j}^{(k)}\right)\right|\right)^{p_{k}} d \mu_{k}\right)^{\frac{p}{p_{k}}} \\
& \leq C^{p} \prod_{k=1}^{n}\left(\sup _{\varphi \in B_{X_{k}^{*}}} \sum_{j=1}^{m}\left(\left|b_{j}^{(k)}\right|\left|\varphi\left(x_{j}^{(k)}\right)\right|\right)^{p_{k}}\right)^{\frac{p}{p_{k}}} .
\end{aligned}
$$

Hence we have (2.1). Conversely, suppose (2.1) and consider the sets $P\left(B_{X_{k}^{*}}\right)$ of the probability measures in $C\left(B_{X_{k}^{*}}\right)^{*}$, for all $k=1, \ldots, n$. It is well-known that each $P\left(B_{X_{k}^{*}}\right)$ is compact when each $C\left(B_{X_{k}^{*}}\right)^{*}$ is endowed with the weak-star topology. For each $\left(x_{j}^{(l)}\right)_{j=1}^{m}$ in $E_{l}$ and $\left(b_{j}^{(s)}\right)_{j=1}^{m}$ in $\mathbb{K}$, with 


$$
\begin{aligned}
(s, l) \in\{1, \ldots, n\} \times\{1, \ldots, n\}, \text { let } & \\
g & =g_{\left(x_{j}^{(l)}\right)_{j=1}^{m},\left(b_{j}^{(s)}\right)_{j=1}^{m},(s, l) \in\{1, \ldots, n\} \times\{1, \ldots, n\}}: P\left(B_{X_{1}^{*}}\right) \times \cdots \times P\left(B_{X_{n}^{*}}\right) \rightarrow \mathbb{R} \\
g\left(\left(\mu_{i}\right)_{i=1}^{n}\right) & =\sum_{j=1}^{m}\left[\frac{1}{p} f\left(b_{j}^{(k)}, x_{j}^{(k)}\right)_{k=1}^{n}-C^{p} \sum_{k=1}^{n} \frac{1}{p_{k}} \int_{B_{X_{k}^{*}}}\left(\left|b_{j}^{(k)}\right|\left|\varphi\left(x_{j}^{(k)}\right)\right|\right)^{p_{k}} d \mu_{k}\right] .
\end{aligned}
$$

Note that the family $\mathcal{F}$ of all such $g$ is concave. In fact, let $N$ be a positive integer, $g_{k} \in \mathcal{F}$ and $\alpha_{k} \geq 0$, $k=1, \ldots, N$, so that $\alpha_{1}+\ldots+\alpha_{N}=1$. We have

$$
\sum_{k=1}^{N} \alpha_{k} g_{k}\left(\left(\mu_{i}\right)_{i=1}^{n}\right) \leq g_{\left(x_{j_{k}}^{(l)}\right)_{j_{k}, k=1}^{m_{k}, N},\left(\alpha_{k}^{\frac{1}{p_{s}}} b_{j_{k}}^{(s)}\right)_{j_{k}, k=1}^{m_{k}, N},(s, l) \in\{1, \ldots, n\} \times\{1, \ldots, n\}}\left(\left(\mu_{i}\right)_{i=1}^{n}\right) .
$$

One can also easily prove that each $g \in \mathcal{F}$ is convex and continuous. Besides, for each $g \in \mathcal{F}$ there are measures $\mu_{k}^{g} \in P\left(B_{X_{k}^{*}}\right), k=1, \ldots, n$, so that

$$
g\left(\mu_{1}^{g}, \ldots, \mu_{n}^{g}\right) \leq 0
$$

In fact, since each $B_{X_{k}^{*}}$ is compact $(k=1, \ldots, n)$ there are $\varphi_{k} \in B_{X_{k}^{*}}$ so that

$$
\sum_{j=1}^{m}\left(\left|b_{j}^{(k)}\right|\left|\varphi_{k}\left(x_{j}^{(k)}\right)\right|\right)^{p_{k}}=\sup _{\varphi \in B_{X_{k}^{*}}} \sum_{j=1}^{m}\left(\left|b_{j}^{(k)}\right|\left|\varphi\left(x_{j}^{(k)}\right)\right|\right)^{p_{k}} .
$$

Now, consider the Dirac measures $\mu_{k}^{g}=\delta_{\varphi_{k}}, k=1, \ldots, n$, and hence

$$
\begin{aligned}
& g\left(\mu_{1}^{g}, \ldots, \mu_{n}^{g}\right)=\sum_{j=1}^{m}\left[\frac{1}{p} f\left(b_{j}^{(k)}, x_{j}^{(k)}\right)_{k=1}^{n}\right]-C^{p} \sum_{k=1}^{n} \frac{1}{p_{k}} \int_{B_{X_{k}^{*}}} \sum_{j=1}^{m}\left(\left|b_{j}^{(k)}\right|\left|\varphi\left(x_{j}^{(k)}\right)\right|\right)^{p_{k}} d \mu_{k}^{g} \\
& =\sum_{j=1}^{m}\left[\frac{1}{p} f\left(b_{j}^{(k)}, x_{j}^{(k)}\right)_{k=1}^{n}\right]-C^{p} \sum_{k=1}^{n} \frac{1}{p_{k}}\left[\left(\sup _{\varphi \in B_{X_{k}^{*}}} \sum_{j=1}^{m}\left(\left|b_{j}^{(k)}\right|\left|\varphi\left(x_{j}^{(k)}\right)\right|\right)^{p_{k}}\right)^{\frac{1}{p_{k}}}\right]^{p_{k}} \\
& \quad \stackrel{(*)}{\leq} \sum_{j=1}^{m}\left[\frac{1}{p} f\left(b_{j}^{(k)}, x_{j}^{(k)}\right)_{k=1}^{n}\right]-C^{p} \frac{1}{p} \prod_{k=1}^{n}\left[\left(\sup _{\varphi \in B_{X_{k}^{*}}} \sum_{j=1}^{m}\left(\left|b_{j}^{(k)}\right|\left|\varphi\left(x_{j}^{(k)}\right)\right|\right)^{p_{k}}\right)^{\frac{1}{p_{k}}}\right]^{p} \\
& \quad{ }^{(* *)} \leq 0
\end{aligned}
$$

where in $\left(^{*}\right)$ we have used Lemma 2.2 and in $\left(*^{*}\right)$ we invoked (2.1). So Ky Fan Lemma applies and we obtain $\overline{\mu_{k}} \in P\left(B_{X_{k}^{*}}\right), k=1, \ldots, n$, so that

$$
g\left(\overline{\mu_{1}}, \ldots, \overline{\mu_{n}}\right) \leq 0
$$

for all $g \in \mathcal{F}$. Hence

$$
\sum_{j=1}^{m}\left[\frac{1}{p} f\left(b_{j}^{(k)}, x_{j}^{(k)}\right)_{k=1}^{n}\right]-C^{p} \sum_{k=1}^{n} \frac{1}{p_{k}} \int_{B_{X_{k}^{*}}} \sum_{j=1}^{m}\left(\left|b_{j}^{(k)}\right|\left|\varphi\left(x_{j}^{(k)}\right)\right|\right)^{p_{k}} d \overline{\mu_{k}} \leq 0
$$


and making $m=1$ we get (for every $b^{(k)} \in \mathbb{K}$ and $x^{(k)} \in X_{k}, k=1, \ldots, n$ )

$$
\begin{aligned}
& \frac{1}{p}\left(\left|b^{(1)} \ldots b^{(n)}\right|\left\|f\left(a_{1}+x^{(1)}, \ldots, a_{n}+x^{(n)}\right)-f\left(a_{1}, \ldots, a_{n}\right)\right\|\right)^{p} \\
& \leq C^{p} \sum_{k=1}^{n} \frac{1}{p_{k}} \int_{B_{X_{k}^{*}}}\left(\left|b^{(k)}\right|\left|\varphi\left(x^{(k)}\right)\right|\right)^{p_{k}} d \overline{\mu_{k}} .
\end{aligned}
$$

Let $x^{(1)}, \ldots, x^{(n)}$ and $b^{(1)}, \ldots, b^{(n)} \neq 0$ be given and, for $k=1, \ldots, n$, define

$$
\tau_{k}:=\left(\int_{B_{X_{k}^{*}}}\left(\left|b^{(k)}\right|\left|\varphi\left(x^{(k)}\right)\right|\right)^{p_{k}} d \overline{\mu_{k}}\right)^{1 / p_{k}} .
$$

If $\tau_{k}=0$ for every $k$ then, from (2.2) we conclude that

$$
\left(\left|b^{(1)} \ldots b^{(n)}\right|\left\|f\left(a_{1}+x^{(1)}, \ldots, a_{n}+x^{(n)}\right)-f\left(a_{1}, \ldots, a_{n}\right)\right\|\right)^{p}=0
$$

and we obtain (1.3), as planned. Let us now suppose that $\tau_{j}$ is not zero for some $j \in\{1, \ldots, n\}$. Consider

$$
V=\left\{j \in\{1, . ., n\} ; \tau_{j} \neq 0\right\}
$$

and for each $\beta>0$ define

$$
\vartheta_{\beta, j}=\left\{\begin{array}{c}
\left(\tau_{j} \beta^{\frac{1}{p p_{j}}}\right)^{-1} \text { if } j \in V \\
1 \text { if } j \notin V .
\end{array}\right.
$$

So, from (2.2), we have

$$
\begin{aligned}
\frac{1}{p} f\left(\vartheta_{\beta, k} b^{(k)}, x^{(k)}\right)_{k=1}^{n} & \leq C^{p} \sum_{k=1}^{n} \frac{1}{p_{k}} \int_{B_{X_{k}^{*}}}\left(\left|\vartheta_{\beta, k} b^{(k)}\right|\left|\varphi\left(x^{(k)}\right)\right|\right)^{p_{k}} d \overline{\mu_{k}} \\
& \leq C^{p} \sum_{k \in V} \frac{1}{p_{k}} \vartheta_{\beta, k}^{p_{k}} \int_{B_{X_{k}^{*}}}\left(\left|b^{(k)}\right|\left|\varphi\left(x^{(k)}\right)\right|\right)^{p_{k}} d \overline{\mu_{k}} \\
& \leq C^{p} \sum_{k \in V} \frac{1}{p_{k}}\left(\tau_{k} \beta^{\frac{1}{p_{k}}}\right)^{-p_{k}} \tau_{k}^{p_{k}} \\
& =C^{p} \sum_{k \in V} \frac{1}{p_{k}} \frac{1}{\beta^{\frac{1}{p}}} \\
& \leq \frac{C^{p}}{p} \frac{1}{\beta^{\frac{1}{p}}} .
\end{aligned}
$$

Hence

$$
\vartheta_{\beta, 1}^{p} \ldots \vartheta_{\beta, n}^{p} \frac{1}{p} f\left(b^{(k)}, x^{(k)}\right)_{k=1}^{n} \leq \frac{C^{p}}{p} \frac{1}{\beta^{1 / p}}
$$


and we have

$$
\begin{aligned}
f\left(b^{(k)}, x^{(k)}\right)_{k=1}^{n} & \leq C^{p} \beta^{-1 / p}\left(\vartheta_{\beta, 1}^{p} \ldots \vartheta_{\beta, n}^{p}\right)^{-1} \\
& =C^{p} \beta^{-1 / p} \prod_{j \in V}\left(\tau_{j} \beta^{\frac{1}{p p_{j}}}\right)^{p} \\
& =C^{p} \beta^{\left(\sum_{j \in V^{1 / p_{j}}}\right)-1 / p} \prod_{j \in V} \tau_{j}^{p} .
\end{aligned}
$$

If $V \neq\{1, \ldots, n\}$, then

$$
\frac{1}{p}-\sum_{j \in V} \frac{1}{p_{j}}>0
$$

Letting $\beta \rightarrow \infty$ in (2.3) we get

$$
f\left(b^{(k)}, x^{(k)}\right)_{k=1}^{n}=0
$$

and we again reach (1.3). If $V=\{1, \ldots, n\}$, from (2.3) we conclude the proof, since

$$
\left(\left|b^{(1)} \ldots b^{(n)}\right|\left\|f\left(a_{1}+x^{(1)}, \ldots, a_{n}+x^{(n)}\right)-f\left(a_{1}, \ldots, a_{n}\right)\right\|\right)^{p}=f\left(b^{(k)}, x^{(k)}\right)_{k=1}^{n} \leq C^{p} \prod_{j=1}^{n} \tau_{j}^{p} .
$$

Note that inequality (2.1) seems to arise an idea of weighted summability. We interpret as each $x_{j}^{(k)}$ has a "weight" $b_{j}^{(k)}$ and in this context the respective sum

$$
\left\|f\left(a_{1}+x_{j}^{(1)}, \ldots, a_{n}+x_{j}^{(n)}\right)-f\left(a_{1}, \ldots, a_{n}\right)\right\|
$$

inherits a weight $\left|b_{j}^{(1)} \ldots b_{j}^{(n)}\right|$. It is easy to note that if $f$ is $n$-linear and $a_{1}=\ldots=a_{n}=0$, then inequality (2.1) coincides with the usual non-weighted inequality. So, the concept of weighted summability can be viewed as a natural extension of the multilinear concept to nonlinear (non-multilinear) maps.

Acknowledgement. The authors thank G. Botelho and P. Rueda for helpful conversations on the topics of this paper. The authors also thank the referee for important suggestions.

\section{REFERENCES}

[1] D. Achour and L. Mezrag, On the Cohen strongly p-summing multilinear operators, J. Math. Anal. Appl. 327 (2007), 550-563.

[2] F. Albiac and N. Kalton, Topics in Banach Space Theory, Springer Verlag, 2006.

[3] H. F. Bohnenblust and E. Hille, On the absolute convergence of Dirichlet series, Ann. Math. 32 (1931), 600-622.

[4] G. Botelho, D. Pellegrino and P. Rueda, Pietsch's factorization theorem for dominated polynomials, J. Funct. Anal 243 (2007), 257-269.

[5] G. Botelho, D. Pellegrino and P. Rueda, A nonlinear Pietsch Domination Theorem, Monatsh. Math. 158 (2009), 247-257.

[6] G. Botelho, D. Pellegrino and P. Rueda, A unified Pietsch Domination Theorem, J. Math. Anal. Appl. 365 (2010), 269-276.

[7] G. Botelho, D. Pellegrino and P. Rueda, Cotype and absolutely summing linear operators. Math. Z., to appear.

[8] W.J. Davis and W.B. Johnson, Compact non-nuclear operators, Studia Math. 51 (1974), 81-85. 
[9] A. Defant and K. Floret, Tensor Norms And Operator Ideals, North-Holland, Amsterdam, 1992.

[10] A. Defant and D. Pérez-García, A tensor norm preserving unconditionality in $L_{p}$-spaces. Trans. Amer. Math. Soc. 360 (2008), 3287-3306.

[11] A. Defant, D. García, M. Maestre and D. Pérez-García, Bohr's strip for vector valued Dirichlet series, Math. Ann. 342 (2008), 533-555.

[12] A. Defant and P. Sevilla-Peris, A new multilinear insight on Littlewood's 4/3-inequality, J. Funct. Anal. 256 (2009), 1642-1664.

[13] A. Defant, D. Popa and U. Schwarting, Coordinatewise multiple summing operators in Banach spaces, J. Funct. Anal 259 (2010), 220-242.

[14] J. Diestel, H. Jarchow, A. Tonge, Absolutely summing operators, Cambridge University Press 1995.

[15] J. Farmer and W. B. Johnson, Lipschitz p-summing operators, Proc. Amer. Math. Soc. 137 (2009), $2989-2995$.

[16] S. Geiss, Ideale multilinearer Abbildungen, Diplomarbeit, 1985.

[17] G. H. Hardy, J.E. Littlewood and G. Polya, Inequalities, Cambridge University Press, 1952.

[18] H. Junek, M. C. Matos and D. Pellegrino, Inclusion theorems for absolutely summing holomorphic mappings, Proc. Amer. Math. Soc. 136 (2008), 3983-3991.

[19] J. Lindenstrauss and A. Pełczyński, Absolutely summing operators in $L_{p}$ spaces and their applications, Studia Math. 29 (1968), 275-326.

[20] J.E. Littlewood, On bounded bilinear forms in an infinite number of variables, Quart. J. (Oxford Ser.) 1 (1930), 164-174.

[21] F. Martínez-Giménez and E. A. Sánchez-Pérez, Vector measure range duality and factorizations of $(D, p)$-summing operators from Banach function spaces, Bull. Braz. Math. Soc. New series 35 (2004), 51-69.

[22] M. C. Matos, Nonlinear absolutely summing multilinear mappings between Banach spaces, Math. Nachr. 258 (2003), 71-89.

[23] M. C. Matos and D. Pellegrino, Fully summing mappings between Banach spaces, Studia Math. 178 (2007), 47-61.

[24] D. Pellegrino, Cotype and absolutely summing homogeneous polynomials in $\mathcal{L}_{p}$ spaces. Studia Math. 157 (2003), 121-231.

[25] D. Pérez-García, Operadores multilineales absolutamente sumantes, Dissertation, Universidad Complutense de Madrid, 2002.

[26] D. Pérez-García, The inclusion theorem for multiple summing operators. Studia Math. 165 (2004), $275-290$.

[27] D. Pérez-García, M.M. Wolf, C. Palazuelos, I. Villanueva and M. Junge, Unbounded violations of tripartite Bell inequalities, Commun. Math. Phys. 279 (2008), 455-486.

[28] A. Pietsch, Absolut p-summierende Abbildungen in normieten Räumen, Studia Math. 27 (1967), 333-353.

[29] A. Pietsch, Operator Ideals, North-Holland, 1980.

[30] R. Ryan, Introduction to Tensor Products of Banach Spaces. Springer Monographs in Mathematics, Springer-Verlag, 2002 .

(D. Pellegrino) Departamento de Matemática, Universidade Federal da Paraíba, 58.051-900 - João Pessoa, Brazil, [J. Santos] Departamento de Matemática, Universidade Federal de Sergipe, 49500-000ITABAIANA, BRAZIL

E-mail address: dmpellegrino@gmail.com 